\title{
Guest editorial for the special issue for Jacques Wolfmann
}

\author{
Yves Aubry • Claude Carlet • \\ Philippe Langevin • Pascal Véron
}

Received: 30 June 2011 / Accepted: 18 July 2011 / Published online: 16 August 2011

(C) Springer Science+Business Media, LLC 2011

This special issue honors Jacques Wolfmann for his entire career. Jacques Wolfmann was born in 1937 in Paris, France. After completing his university studies in mathematics, he received the "Agrégation" degree in mathematics in 1970. During this period, he was also a teacher at the University of Quebec in Canada (1969-1971). He defended a PhD thesis under the supervision of Paul Camion in 1973, and was awarded the "Doctorat d'Etat" degree in coding theory, by the University of Paris 7 in 1978. In 1981 he became a professor at France's University of Toulon.

He has worked in various fields such as coding theory, combinatorics, algebraic geometry and number theory. He has published nearly 50 papers on sequences, cyclic codes, difference sets, codes over $\mathbb{Z}_{4}$. Using coding theory he obtained several results on Kloosterman sums and elliptic curves, on the number of rational points of some algebraic curves, on diagonal equations over finite fields and on bent functions.

In 1981 at the University of Toulon, he created a coding theory research team called GECT, one of the first laboratories in France in this research area. In 2000, it became the GRIM Laboratory, a research team in computer science and mathematics which Jacques Wolfmann headed for several years.

As a teacher, Jacques is an outstanding educator, contributing greatly to the development of discrete mathematics, and specifically, to coding theory in France. Notably, he was vice-president of the "Société Mathématique de France" from 2003 to 2006 .

At an international level, he is a member of the editorial boards of "Applicable Algebra in Engineering Communication and Computing", "Finite Fields and Their Applications" and "Designs, Codes and Cryptography".

Y. Aubry · P. Langevin · P. Véron ( $ه)$

Université du Sud Toulon-Var, 83957 La Garde cedex, France

e-mail: veron@univ-tln.fr

C. Carlet

Université Paris 8, 93526, Saint-Denis cedex 02, France 
In addition to mathematics, music plays an important role in the life of Jacques, especially classical and barocco style. He has been conducting the University Choir of Toulon for many years and performed numerous concerts.

At present, Jacques Wolfmann is "Professeur émérite" at the "Université du Sud Toulon-Var" IMATH Institute of Mathematics.

One motivating event for this volume was the YACC international conference in Cryptography held in his honor in Porquerolles Island (France) in October 2010. This conference is organized by the IMATH institute every 2 years.

Some of the authors herein were speakers at this conference. They gave major contributions in some of Jacques Wolfmann's favorite research topics. Eight papers have been collected for this issue. Stéphane Ballet and Robert Rolland revisit Yao's theorem on pseudo-random generators in terms of probabilistic computational complexity. John Dillon demonstrates a new proof about the dimension of the codes associated with APN functions. Tor Helleseth and Alexander Kolosha write a survey article relating bent functions, m-sequences and Jacobsthal sums. Marc Mouffron and Christelle Rovetta study the representation of multivalued functions with Multivalued Decision Diagrams. Jérémy Parriaux, Philippe Guillot and Gilles Millérioux propose a new approach to the building of self-synchronizing stream ciphers. Alexander Pott and Yue Zhou present a characterization of planar function using character theory. Francois Rodier exhibits a family of functions which are not APN infinitely often. Finally, Elif Saygi, Zülfükar Saygi and Ferruh Özbudak give a full classification of quadratic forms in characteristic 2 of the form $x \mapsto \operatorname{Tr}(x L(x))$ where $L(x)$ are linearized polynomials with three coefficients in $\mathbf{F}_{4}$.

We thank Springer for approving this special issue as well as our referees for the time spent during the submission process. 